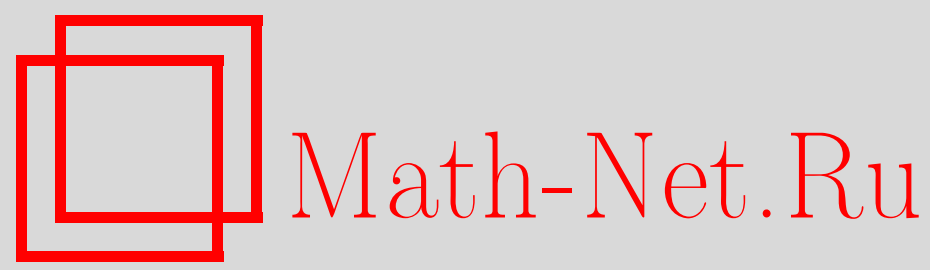

Н. А. Бобылев, Несколько замечаний о звездных множествах, Матем. заметки, 1999, том 65, выпуск 4, 511-519

DOI: https://doi.org/10.4213/mzm1077

Использование Общероссийского математического портала Math-Net.Ru подразумевает, что вы прочитали и согласны с пользовательским соглашением http://www. mathnet.ru/rus/agreement

Параметры загрузки:

IP : 54.147 .182 .235

26 апреля 2023 г., 18:18:00

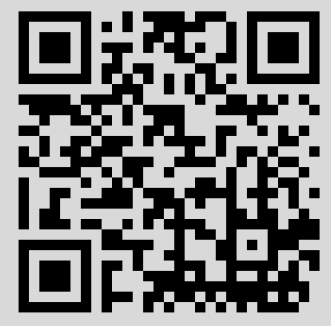




\title{
НЕСКОЛЬКО ЗАМЕЧАНИЙ О ЗВЕЗДНЫХ МНОЖЕСТВАХ
}

\author{
Н. А. Бобылев
}

В работе рассматриваются вопросы, связанные со свойствами объединений и пересечений звездных компактов в конечномерных пространствах.

Библиография: 7 названий.

1. Основные определения. Через $E^{N}$ обозначим $N$-мерное вешественное евклидово пространство векторов $x=\left\{x_{1}, \ldots, x_{N}\right\}$; через $B(r, x)$ - шар радиуса $r$ с центром в точке $x$ :

$$
B(r, x)=\left\{y \in E^{N}:\|y-x\| \leqslant r\right\}
$$

через $S(r, x)$ - сферу радиуса $r$ с центром в точке $x$ :

$$
S(r, x)=\left\{y \in E^{N}:\|y-x\|=r\right\} .
$$

Через conv $M$ обозначим выпуклую оболочку множества $M$, т.е. минимальное (по включению) выпуклое множество, содержащее $M$. Через $\operatorname{card} M$ обозначим мощность множества $M$. В частности, если $M$ состоит из $n$ элементов, то card $M=n$.

Пусть $x \in E^{N}$ - некоторая точка. Через $d_{M}(x)$ обозначим расстояние точки $x$ до множества $M$ :

$$
d_{M}(x)=\inf _{y \in M}\|x-y\|
$$

Непустое множество $M \subset E^{N}$ назьвается звездным, если существует точка $x_{*} \in M$, из которой “видны" в $M$ остальные точки этого множества, т.е. для любой точки $x \in M$ отрезок $\left[x_{*}, x\right]=\left\{y \in E^{N}: y=(1-\lambda) x_{*}+\lambda x, 0 \leqslant \lambda \leqslant 1\right\}$ лежит в $M$.

Множество всех точек звездного множества $M$, из которых видны остальные точки этого множества, назьвается звездой $s(M)$ множества $M$. Звезда звездного множества является выпуклым множеством.

Множество $\mathscr{K} \subset E^{N}$ назьвается конусом, если для каждой точки $x \in \mathscr{K}$ и числа $\lambda \in[0, \infty)$

$$
\lambda x \in \mathscr{K}
$$

Работа вьполнена в рамках Международного проекта СТIAC при поддержке Российского фонда фундаментальных исследований, гранты № 98-01-00586 и № 99-01-00883. 
Из этого определения следует, что конус - звездное множество $0 \in s(\mathscr{K})$. Конус $\mathscr{K}$ назьвается миниәдральным, если непустое пересечение транслятов

$$
\mathscr{K}_{x}=\left\{y \in E^{N}: y=z+x, z \in \mathscr{K}\right\}
$$

конуса $\mathscr{K}$ является транслятом этого конуса.

Пусть $K \subset E^{N}-$ произвольньй компакт и $\mathscr{K} \subset E^{N}-$ замкнутьй миниэдральньй выпукльй конус. Семейство $K_{x}(x \in K)$ лежащих в $K$ звездных компактов назовем $\mathscr{K}$-правильным, если оно обладает следующими свойствами:

1) каждая точка $x \in K$ принадлежит звезде $s\left(K_{x}\right)$ компакта $K_{x}$;

2) $K_{x} \subset \mathscr{K}_{x}(x \in K)$;

3) для каждых $x, y \in K$ таких, что $x \neq y,[x, y] \subset \mathscr{K}_{x}$, справедливо включение

$$
l_{[x, y]} \subset K_{x}
$$

где $l_{[x, y]}$ - максимальный замкнутый отрезок, лежаший в $K$, одним из концов которого является точка $x$ и которьй содержит точку $y$.

2. Критерии звездности. В построениях этого пункта будем использовать теорему Хелли о непустоте пересечения семейства вьпуклых компактов. Приведем ее формулировку.

ТЕорема 1 (Хелли [1]). Пусть $C_{i}(i \in I)$ - семейство непустых выпуклых компактов в $E^{N}$. Если для каждых $N+1$ компактов $C_{i_{1}}, \ldots, C_{i_{N+1}}$ этого семейства

$$
\bigcap_{j=1}^{N+1} C_{i_{j}} \neq \varnothing
$$

mo

$$
\bigcap_{i \in I} C_{i} \neq \varnothing
$$

Доказательство теоермы Хелли, а также ее многочисленные обобщения и применения содержатся в мнографии [2].

Рассмотрим некоторый компакт $K \subset E^{N}$.

ЛЕмма 1. Пусть для некоторого К-правильного семейства звездных компакmoв $K_{x}(x \in K)$

$$
\bigcap_{x \in K} \operatorname{conv} K_{x} \neq \varnothing
$$

Тогда

$$
\bigcap_{x \in K} K_{x} \neq \varnothing .
$$


ДокАЗАТЕЛЬСТво. Предположим противное, т.е.

$$
\bigcap_{x \in K} K_{x}=\varnothing .
$$

Пусть

$$
y_{0} \in \bigcap_{x \in K} \operatorname{conv} K_{x} .
$$

Тогда найдется точка $x_{0} \in K$, для которой $y_{0} \notin K_{x_{0}}$. Рассмотрим на отрезке $\left[x_{0}, y_{0}\right]$ непрерьвную функцию $d_{K}(\cdot)$ и через $a$ обозначим максимум этой функции на $\left[x_{0}, y_{0}\right]$. Очевидно, $a>0$. Обозначим через $x_{1}$ какую-нибудь из точек отрезка $\left[x_{0}, y_{0}\right]$, для которой $d_{K}\left(x_{1}\right)=a$, а через $A \subset K$ множество тех $y \in K$, для которых $\left\|x_{1}-y\right\|=a$. Проведем через каждую точку $y \in A$ гиперплоскость $H_{y}$ ортогональную вектору $y-x_{1}$ и покажем, что для некоторого $y_{1} \in A$ точки $x_{1}$ и $y_{0}$ лежат в одном из полупространств, на которые гиперплоскость $H_{y_{1}}$ делит $E^{N}$.

Для этого рассмотрим сферу $S\left(a, x_{1}\right)$. Согласно определению точки $x_{1}$ и числа $a$

$$
\operatorname{int} B\left(a, x_{1}\right) \cap K=\varnothing, \quad S\left(a, x_{1}\right) \cap K=A .
$$

Далее, рассмотрим гиперплоскость $H$, проходящую через точку $x_{1}$ и ортогональную вектору $x_{0}-y_{0}$. Пусть $\Pi_{0}$ и $\Pi_{1}-$ полупространства, на которые разбивает $E^{N}$ гиперплоскость $H$, причем $x_{0} \in \bar{\Pi}_{0}, y_{0} \in \bar{\Pi}_{1}$. Если

$$
S\left(a, x_{1}\right) \cap \bar{\Pi}_{0} \cap K \neq \varnothing,
$$

то в качестве $y_{1}$ можно взять любую точку последнего пересечения. Если же

$$
S\left(a, x_{1}\right) \cap \bar{\Pi}_{0} \cap K=\varnothing,
$$

то в качестве $y_{1}$ можно взять любую точку множества $A$. Действительно, если это не так, то при малых $\varepsilon>0$ для точек $x_{\varepsilon}=x_{1}+\varepsilon\left(x_{0}-y_{0}\right)$ вьполнено неравенство

$$
d_{K}\left(x_{\varepsilon}\right)>\left\|x_{1}-y_{1}\right\|=a,
$$

что противоречит выбору точки $x_{1}$.

Итак, пусть $y_{1} \in A$ - такая точка множества $A$, для которой гиперплоскость $H_{y_{1}}$, проходящая через $y_{1}$ и ортогональная вектору $y_{1}-x_{1}$, разбивает $E^{N}$ на полупространства $\Pi_{0}$ и $\Pi_{1}$, причем $x_{1}, y_{0} \in \Pi_{0}$. Рассмотрим множество $K_{y_{1}}$. Оно целиком лежит в замыкании полупространства $\Pi_{1}$. Действительно, если некоторая точка $y_{2} \in K_{y_{1}}$ лежит в $\Pi_{0}$, то в силу звездности множества $K_{y_{1}}$ и включения $y_{1} \in s\left(K_{y_{1}}\right)$ отрезок $\left[y_{1}, y_{2}\right]$ лежит в $K_{y_{1}}$ и, следовательно, лежит в $K$. Но тогда для некоторой точки $y_{3} \in\left[y_{1}, y_{2}\right]$

$$
\left\|x_{1}-y_{3}\right\|<\left\|x_{1}-y_{1}\right\|=a .
$$

Поэтому $d_{K}\left(x_{1}\right)<a$, что противоречит определению точки $x_{1}$.

Итак, $K_{y_{1}} \subset \bar{\Pi}_{1}$. Но тогда conv $K_{y_{1}} \subset \bar{\Pi}_{1}$ и, следовательно, $y_{0} \in \operatorname{conv} K_{y_{1}}$. Мы пришли к противоречию. Лемма доказана.

Из леммы 1 вытекает 
ТЕорема 2 (критерий звездности). Пусть для некоторого K-правильного семейства звездных компактов

$$
\bigcap_{x \in K} \operatorname{conv} K_{x} \neq \varnothing .
$$

Тогда компакт $K$ является звездным.

ДокАЗАТЕЛЬСТВо. В силу леммы 1

$$
\bigcap_{x \in K} K_{x} \neq \varnothing .
$$

Пусть $x_{*}-$ произвольная точка последнего пересечения. Согласно свойству 1$)$, которым обладает семейство $K_{x}(x \in K)$, любая точка $x_{0} \in K$ лежит в звезде $s\left(K_{x_{0}}\right)$ звездного компакта $K_{x_{0}} \subset K$. Поскольку $x_{*} \in K_{x_{0}}$, то по определению множества $s\left(K_{x_{0}}\right)$ отрезок $\left[x_{0}, x_{*}\right]$ лежит в $K_{x_{0}}$ и, следовательно, в $K$. Теорема доказана.

Из теоремы 2 вытекает следующий критерий звездности, принадлежаший М. А. Красносельскому.

ТЕОрема 3 (М. А. Красносельский [3]). Пусть для любы $N+1$ точек $x_{1}, \ldots, x_{N+1}$ компакта $K \subset E^{N}$ существует точка $x_{*} \in K$, из которой виднь точки $x_{i}$ $(i=1, \ldots, N+1)$. Тогда компакт $K$ является звездным.

ДокАЗАТЕЛЬСТво. Для каждого $x \in K$ положим

$$
K_{x}=\{y \in K:[x, y] \subset K\}
$$

Очевидно, каждьй компакт $K_{x}$ является звездньп. Из определения семейства $K_{x}$ $(x \in K)$ следует, что оно является $E^{N}$-правильным.

По условию теоремы каждые $N+1$ компактов $K_{x_{1}}, \ldots, K_{x_{N+1}}$ семейства $K_{x}(x \in K)$ имеют непустое пересечение. Но тогда и вьпуклые оболочки conv $K_{x_{1}}, \ldots$, conv $K_{x_{N+1}}$ этих компактов имеют непустое пересечение. Следовательно, по теореме Хелли

$$
\bigcap_{x \in K} \operatorname{conv} M_{x} \neq \varnothing .
$$

Остается сослаться на теорему 2. Теорема доказана.

3. Условие конуса. Пусть $\mathscr{K} \subset E^{N}$ - выпукльй замкнутьй миниэдральный конус. Будем говорить, что звездный компакт $K$ удовлетворяет $\mathscr{K}$-условию, если существует точка $x_{*} \in s(K)$ такая, что для любой точки $x \in K$

$$
x-x_{*} \in \mathscr{K} .
$$

Звездньй компакт $K$ удовлетворяет $\mathscr{K}$-условию тогда и только тогда, когда для некоторой точки $x_{*} \in s(K)$ транслят

$$
\mathscr{K}\left(x_{*}\right)=\left\{y \in E^{N}: y=x+x_{*}, x \in \mathscr{K}\right\}
$$

конуса $\mathscr{K}$ содержит $K . \mathscr{K}$-условие характеризует расположение звездного компакта $K$ в $E^{N}$. Лемма 1 , установленная в предыдущем пункте, дает критерий $\mathscr{K}$-условия для компакта $K$. 
Теорема 4. Пусть для каждыих $N+1$ точек $x_{1}, \ldots, x_{N+1} \in K$ существует такая точка $x_{0}$, из которой видны әти точки в множестве $K \cap \mathscr{K}\left(x_{0}\right)$. Тогда компакт $K$ удовлетворяет $\mathscr{K}$-условию.

ДокАЗАТЕЛЬСТво. Для каждой точки $x \in K$ через $K_{x}$ обозначим множество всех таких $y \in K$, которые видны из $x$ в $K \cap(-\mathscr{K}(x))$. Семейство компактов $K_{x}$ является $K$-правильньм. По условию теоремы каждые $N+1$ компактов $K_{x_{1}}, \ldots, K_{x_{N+1}}$ имеют непустое пересечение. Таким образом,

$$
\bigcap_{i=1}^{N+1} \operatorname{conv} K_{x_{i}} \neq \varnothing
$$

и по теореме Хелли

$$
\bigcap_{x \in K} \operatorname{conv} K_{x} \neq \varnothing
$$

Значит, в силу леммы 1

$$
\bigcap_{x \in K} K_{x} \neq \varnothing
$$

Пусть

$$
x_{*} \in \bigcap_{x \in K} K_{x} .
$$

Тогда по теореме $2 x_{*} \in s(K)$ и $K \subset \mathscr{K}\left(x_{*}\right)$. Теорема доказана.

4. Теорема Хелли для звездных компактов. Непустой компакт в $E^{N}$ называется гомологической клеткой, если он гомологически тривиален во всех размерностях (см., например, [4]). Для гомологических клеток справедлива вторая теорема Хелли.

Теорема 5 (Хелли [5]). Пусть $K_{i}(i \in I)$ - семейство гомологических клеток в $E^{N}$. Пусть для каждого подмножества $K_{i}\left(i \in I_{0}\right)$ этого семейства $c$ card $I_{0} \leqslant$ $N+1$ пересечение

$$
\bigcap_{i \in I_{0}} K_{i}
$$

является гомологической клеткой. Тогда

$$
\bigcap_{i \in I} K_{i}
$$

является гомологической клеткой.

Комбинаторное доказательство теоремы Хелли содержится в монографии П. С. Александрова и Х. Хопфа [6].

Важный класс гомологических клеток составляют звездные компакты. Применение теоремы 5 к семейству звездных компактов в $E^{N}$ приводит к следующему результату. 
Теорема 6. Пусть $K_{i}(i \in I)$ - семейство звездных компактов в $E^{N}$ и для каждого подмножества $K_{i}\left(i \in I_{0}\right)$ этого семейства $c$ card $I_{0} \leqslant N+1$ пересечение

$$
\bigcap_{i \in I_{0}} K_{i}
$$

непусто и звездно. Тогда

$$
\bigcap_{i \in I} K_{i} \neq \varnothing
$$

Теорема 6 хорошо известна и содержится, например, в качестве упражнения в книге [7]. В условиях этой теоремы пересечение всего семейства звездных компактов будет не только не пустым, но и являться гомологической клеткой. Это замечание допускает важное уточнение.

Tеорема 7. Пусть $K_{i}(i \in I)$ - семейство звездных компактов в $E^{N}$ и для каждого подмножества $K_{i}\left(i \in I_{0}\right)$ этого семейства $c$ card $I_{0} \leqslant N+1$ пересечение

$$
\bigcap_{i \in I_{0}} K_{i}
$$

непусто и звездно. Тогда пересечение

$$
\bigcap_{i \in I} K_{i}
$$

непусто и звездно.

ДоКАЗАТЕЛЬСТво. ПоЛожим

$$
K_{*}=\bigcap_{i \in I} K_{i}
$$

В силу теоремы $6 K_{*} \neq \varnothing$. Покажем, что $K_{*}$ звездно.

Пусть $x_{1}, \ldots, x_{N+1} \in K_{*}-$ некоторые точки. Для каждого $i \in I$ через $M_{i}$ обозначим совокупность всех точек $x \in K_{i}$, из которых видны в $K_{i}$ точки $x_{1}, \ldots, x_{N+1}$. Множество $M_{i}$ непусто, так как $s(K) \subset M_{i}$.

Зафиксируем некоторое натуральное $k \leqslant N+1$ и рассмотрим произвольные множества $M_{i_{1}}, \ldots, M_{i_{k}}$. Положим

$$
M_{i_{1} \ldots i_{k}}=\bigcap_{j=1}^{k} M_{i_{j}} .
$$

Покажем, что множество $M_{i_{1} \ldots i_{k}}$ непусто и звездно.

Рассмотрим множество

$$
K_{i_{1} \ldots i_{k}}=\bigcap_{j=1}^{k} K_{i_{j}} .
$$

По условию теоремы множество $K_{i_{1} \ldots i_{k}}$ непусто и звездно. Пусть $x_{0} \in s\left(K_{i_{1} \ldots i_{k}}\right)$. Так как $x_{j} \in K_{i_{1} \ldots i_{k}}(j=1, \ldots, N+1)$, каждая точка $x_{j}$ видна в множестве $K_{i_{1} \ldots i_{k}}$ из точки $x_{0}$. Поэтому $x_{0} \in M_{i_{1} \ldots i_{k}}$ и, следовательно, $M_{i_{1} \ldots i_{k}} \neq \varnothing$. Покажем, что множество $M_{i_{1} \ldots i_{k}}$ звездно и

$$
s\left(K_{i_{1} \ldots i_{k}}\right) \subset s\left(M_{i_{1} \ldots i_{k}}\right) .
$$


Пусть $x \in s\left(K_{i_{1} \ldots i_{k}}\right), y \in M_{i_{1} \ldots i_{k}}$. Рассмотрим произвольную точку $x_{j}(j=1, \ldots$, $N+1)$. Так как $K_{i_{1} \ldots i_{k}}$ - звездное множество, $\left[y, x_{j}\right] \subset K_{i_{1} \ldots i_{k}}, x \in s\left(K_{i_{1} \ldots i_{k}}\right)$, то треугольник с вершинами $x, y, x_{j}$ лежит в $K_{i_{1} \ldots i_{k}}$. Но тогда точка $x_{j}$ видна в множестве $K_{i_{1} \ldots i_{k}}$ из каждой точки отрезка $[x, y]$. Таким образом, все точки $x_{1}, \ldots, x_{N+1}$ видны из любой точки отрезка $[x, y]$. Поэтому

$$
[x, y] \subset M_{i_{1} \ldots i_{k}}
$$

и, следовательно, $M_{i_{1} \ldots i_{k}}-$ звездное множество, причем $s\left(K_{i_{1} \ldots i_{k}}\right) \subset s\left(M_{i_{1} \ldots i_{k}}\right)$ В силу теоремы 6

$$
M_{*}=\bigcap_{i \in I} M_{i} \neq \varnothing .
$$

Пусть $x_{*} \in M_{*}$. Так как $M_{*} \subset K_{*}$, то $x_{*} \in K_{*}$. По определению множеств $M_{i}(i \in I)$ из точки $x_{*}$ видны в множестве $K_{*}$ все точки $x_{1}, \ldots, x_{N+1}$. Тогда в силу теоремы 2 множество $K_{*}$ звездно. Теорема доказана.

5. Двойственная теорема. Теоремы Хелли 1 и 5 и родственные им утверждения являются инструментами исследования пересечений семейств множеств. В связи с этими утверждениями представляют интерес двойственные утверждения о свойствах объединений семейств множеств. В этом пункте приводится теорема, являющаяся двойственной к теореме 7.

TЕОрема 8. Пусть $K_{i}(i \in I)$ - семейство звездных компактов в $E^{N} u$ card $I \geqslant$ $N+1$. Пусть для каждого $k \leqslant N+1$ объединение любых $k$ компактов этого семейства звездно. Тогда обвединение $K^{*}$ всех компактов семейства звездно.

ДокАЗАТЕЛЬСТво. Пусть $x_{1}, \ldots, x_{N+1}-$ произвольные точки компакта

$$
K^{*}=\bigcup_{i \in I} K_{i} .
$$

Все точки $x_{1}, \ldots, x_{N+1}$ лежат в объединении не более чем $N+1$ компактов рассматриваемого семейства. Обозначим их через $K_{i_{1}}, \ldots, K_{i_{k}}(k \leqslant N+1)$. По условию теоремы объединение

$$
\bigcup_{j=1}^{l} K_{i_{j}}
$$

звездно. Поэтому найдется точка $x_{*} \in K^{*}$ такая, что отрезки $\left[x_{*}, x_{1}\right], \ldots,\left[x_{*}, x_{N+1}\right]$ лежат в последнем объединении и, следовательно, в $K$. Остается сослаться на теорему Красносельского. Теорема доказана.

6. Звездность в задачах о пересечениях. Свойство звездности можно использовать в задачах о пересечениях выпуклых множеств. Приведем один результат в этом направлении.

ТЕорема 9. Пусть $C_{i}(i \in I)$ - конечное семейство выпуклых компактов в $E^{N}$ $u$ card $I \geqslant N+1$. Пусть для кажсдого $k \leqslant N+1$ облединение любых $k$ компактов этого семейства звездно. Тогда

$$
\bigcap_{i \in I} C_{i} \neq \varnothing .
$$

Доказательству теоремы предпошлем два вспомогательных утверждения. 
Лемма 2 (Кли [2]). Пусть $C_{1}, \ldots, C_{N+1}$ - выпуклые компакты в $E^{N}$, оббединение которых выпукло. Пусть каждые $N$ компактов этого семейства имеют непустое пересечение. Тогда

$$
\bigcap_{i=1}^{N+1} C_{i} \neq \varnothing .
$$

Лемма 3. Пусть $C_{1}, \ldots, C_{n}-$ выпуклве компакты в $E^{N} u n \leqslant N+1$. Пусть для каждого $k \leqslant n$ обгединение любы $k$ компактов этого семейства звездно. Тогда

$$
\bigcap_{i=1}^{n} C_{i} \neq \varnothing
$$

ДокАЗАТЕЛЬСТво проведем индукцией по $n$. Для $n=1$ утверждение леммы очевидно. Пусть утверждение леммы справедливо для любого семейства вьпуклых компактов $C_{i}(i \in I)$, если $\operatorname{card} I \leqslant n$ и $n \leqslant N$. Докажем лемму для семейства компактов $C_{1}, \ldots, C_{n+1}$. Положим

$$
\mathscr{D}_{i}=\bigcap_{j \neq i} C_{j}, \quad i=1, \ldots, n+1 .
$$

По предположению индукции множества $\mathscr{D}_{i}$ непусты. Выберем в каждом множестве $\mathscr{D}_{i}$ точку $x_{i}$. Без ограничения общности можно считать, что точки $x_{i}(i=1, \ldots, n+1)$ находятся в общем положении. Рассмотрим $n$-мерньй симплекс

$$
\Delta_{n}=\operatorname{conv}\left\{x_{1}, \ldots, x_{n+1}\right\}
$$

и положим

$$
M_{i}=\mathscr{D}_{i} \cap \Delta_{n}, \quad i=1, \ldots, n+1 .
$$

Так как множество $M_{i}$ содержит $(n-1)$-мерную грань $\operatorname{conv}\left\{x_{1}, \ldots, x_{i-1}\right.$, $\left.x_{i+1}, \ldots, x_{n+1}\right\}$, граница $\partial \Delta_{n}$ симплекса $\Delta_{n}$ содержится в объединении множеств $M_{i}$ $(i=1, \ldots, n+1)$ :

$$
\partial \Delta_{n} \subset \bigcup_{i=1}^{n+1} M_{i} .
$$

Но тогда

$$
\Delta_{n}=\bigcup_{i=1}^{n+1} M_{i} .
$$

Действительно, если равенство (1) не выполнено, то граница $\partial \Delta_{n}$ симплекса $\Delta_{n}$ содержится в объединении $\mathscr{D}$ множеств $\mathscr{D}_{i}(i=1, \ldots, n+1)$ :

$$
\partial \Delta_{n} \subset \bigcup_{i=1}^{n+1} \mathscr{D}_{i},
$$

а некоторая точка $x_{0} \in \operatorname{int} \Delta$ не лежит в $\mathscr{D}$. По условию леммы множество $\mathscr{D}$ звездно. Пусть $x_{*}$-произвольная точка, лежащая в звезде $s(\mathscr{D})$ множества $\mathscr{D}$. Рассмотрим луч $l$ с началом в $x_{*}$, проходящий через точку $x_{0}$. Поскольку $\partial \Delta_{n} \subset \mathscr{D}$, а $x_{0} \notin \mathscr{D}$, то луч $l_{0} \mathrm{c}$ 
вершиной $x_{0}$, направление которого совпадает с направлением $l$, пересекает $\mathscr{D}$ в некоторой точке $y_{0}$. Но отрезок $\left[x_{*}, y_{0}\right]$ не лежит целиком в $\mathscr{D}$, что противоречит звездности $\mathscr{D}$.

Итак, соотношение (1) установлено. Тогда в силу леммы 2

$$
\bigcap_{i=1}^{n+1} C_{i} \neq \varnothing .
$$

Лемма доказана.

ДокАЗАТЕЛЬСТво ТЕОРЕмЫ 9. Рассмотрим произвольные $N+1$ компактов $C_{i_{1}}, \ldots, C_{i_{N+1}}$ семейства $C_{i}(i \in I)$. Семейство компактов $C_{i_{j}}(j=1, \ldots, N)$ удовлетворяет условиям леммы 3 при $n=N+1$. Поэтому

$$
\bigcap_{j=1}^{N+1} C_{i_{j}} \neq \varnothing
$$

Тогда в силу первой теоремы Хелли

$$
\bigcap_{i \in I} C_{i} \neq \varnothing .
$$

Теорема доказана.

В заключение автор выражает благодарность С. М. Ассееву за обсуждение этой работы и полезные замечания.

\section{СПИСОК ЦИТИРОВАННОЙ ЛИТЕРАТУРЫ}

[1] Helly E. Über Mengen Konvexer Körper mit gemeinschaftlichen Punkten // Iber. Deutsch. Math. Verein. 1923. V. 32. P. 175-176.

[2] Данцер Л., Грюнбаум Б., Кли В. Теорема Хелли и ее применения. М.: Мир, 1968.

[3] Красносельский М.А. Об одном критерии звездности // Матем. сб. 1946. Т. 19. C. $309-310$.

[4] Зейферт Т., Трельфалль В. Топология. М.-Л.: ГОНТИ, 1938.

[5] Helly E. Über Systeme abgeschlossener Mengen mit gemeinschaftlichen Punkten // Monatsch. Math. 1930. V. 37. P. 281-302.

[6] Alexandroff P., Hopf H. Topologie. V. 1. Berlin, 1935.

[7] Кириллов А. А., Гвишиани А. Д. Теоремы и задачи функционального анализа. М.: Наука, 1979.

Институт проблем управления РАН

Поступило 08.07 .98

Исправленный вариант 